\title{
Respiratory Syncytial Virus Rhinosinusitis in Intensive Care Unit Patients
}

\author{
Alexandre Rodrigues da Silva ${ }^{1}$, Marcelo Park ${ }^{2}$, Lucy S. Vilas Boas ${ }^{1}$ and Clarisse Martins Machado ${ }^{1}$ \\ ${ }^{1}$ Virology Laboratory (LIM 52-HCFMUSP), Institute of Tropical Medicine, University of São Paulo Medical School; ${ }^{2}$ Intensive Care Unit, \\ Department of Internal Medicine, Hospital das Clínicas, University of São Paulo Medical School; São Paulo, SP Brazil
}

\begin{abstract}
This study reported a case of rhinosinusitis for Respiratory Syncytial Virus in Intensive Care Unit patient. The settings were Intensive Care Unit at Hospital das Clínicas, University of São Paulo Medical School, São Paulo, Brazil. One female HIV-infected patient with respiratory failure and circulatory shock due to splenic and renal abscesses, who developed rhinosinusitis caused by RSV and bacteria. Respiratory viruses can play a pathogenic role in airways infection allowing secondary bacterial overgrowth.
\end{abstract}

Key-Words: Respiratory Syncytial Virus, RSV, rhinosinusitis, AIDS, ICU infection, viral infection.

Rhinosinusitis is one of the most common health complaints. Its incidence varies from 1.6 to 3.5 episodes per 100 adults per year in Western developed countries [1]. In specific groups, such as HIV-infected patients, sinusitis is particularly relevant, and occurs in $20 \%$ to $68 \%$ of them [2].

Respiratory viruses (RV) are a major cause of upper respiratory tract infections (URI). It has been estimated that viral colds lead to the development of bacterial rhinosinusitis in $0.5 \%$ to $2.5 \%$ of adult patients [1,3] and $10 \%$ of children [3].

The use of imaging methods showed that abnormalities in the paranasal sinuses are very frequent during episodes of upper respiratory tract viral infection. Maxillary sinus radiographs of young adults with typical viral upper respiratory tract infections (URIs) showed mucosal abnormalities in 39\% of cases and computed tomographic (CT) scans were abnormal in $87 \%$ of similar cases [1].

Acute bacterial sinusitis has been reported to follow the common cold. Studies performing consecutive cultures of sinus punctures showed that about $60 \%$ of patients with upper RSV infection who remained symptomatic for at least one week, had a positive bacterial aspirate culture later [4].

\section{Case Report}

A 28-year-old female patient with HIV infection diagnosed one year earlier was admitted to hospital with asthenia, progressive muscle weakness, loss of weight in the previous six months and fever in the previous 24 hours. The immunologic and virologic status and previous antiretroviral therapy experience were unknown. She had no previous history of smoking, alcohol or illicit drug addiction.

Three days after admission, her condition deteriorated and she was admitted to the Intensive Care Unit presenting symptoms of fever followed by respiratory failure and circulatory shock. Norepinephrine was started and she

Received on 16 September 2006; revised 2 December 2006.

Address for correspondence: Dr. Clarisse Martins Machado. Av. Dr. Enéas de Carvalho Aguiar, 470. Zip code: 05403-000, São Paulo/SP, Brazil. Financial support used for the study, including any institutional departmental funds: none.

The Brazilian Journal of Infectious Diseases 2007;11(1):163-165. (C) 2007 by The Brazilian Journal of Infectious Diseases and Contexto Publishing. All rights reserved. required mechanical ventilation. Blood cultures were taken and the patient underwent bronchoalveolar lavage (BAL). Trimethoprim/sulfamethoxazole, azithromycin, oxacillin and cefepime were started empirically.

Four days after admission to hospital, methicillinsusceptible Staphylococcus aureus and Streptococcus pneumoniae were identified in the patient's blood culture and BAL culture. RSV was identified in the nasopharyngeal lavage by direct immunofluorescence assay (DFA) and polymerase chain reaction (PCR). Seven days after hospital admission, the nasopharyngeal lavage remained positive for RSV, but turned negative on the eleventh, fourteenth and eighteenth days.

Seven days after admission to hospital, a computed tomographic (CT) scan demonstrated splenic and right renal abscesses, partial thrombosis of the renal vein, bilateral pleural effusion with left pulmonary condensation, and pericardial effusion. Nine days after hospital admission she was submitted to right nephrectomy and splenectomy. Pericardiotomy was performed on the fifth day after hospital admission. Staphylococcus aureus was isolated in cultures of the splenic and renal abscesses.

Eighteen days after hospital admission, nosocomial sinusitis and nosocomial pneumonia were diagnosed and the therapy was switched to vancomycin and imipenem. The left maxillary sinus was aspirated and RSV was identified by DFA and PCR. Multiresistant Acinetobacter baumannii was identified in culture of nasal sinus aspirate.

Despite the use of wide-spectrum antibiotics, after 20 days of hospitalization the patient developed hemodynamic instability and refractory shock and died.

\section{Discussion}

The maxillary sinus of an adult has a volume of approximately 15 to $30 \mathrm{~mL}$, and the diameter of the infundibulum is capable of draining $30 \mathrm{~mL}$ of water from the maxillary sinus by gravity in approximately 11 seconds [5]. During an episode of viral rhinosinusitis, increased amounts of viscous material can be expected to accumulate in the sinuses as a consequence of local inflammation and to decrease movement of the respiratory epithelium cilia, which move the secretions toward the infundibulum ostium for clearance [5]. This allows the overgrowth of bacteria [5]. 
The role of respiratory viruses in the pathogenesis of rhinosinusitis has been postulated, as bacteria have been isolated in only $60 \%$ of sinus aspirates in suspected cases of acute community-acquired sinusitis [4]. However, few studies have searched for respiratory viruses in paranasal sinus aspirates. Hamory et al. analyzed 105 aspirates of the facial sinuses of 81 patients with a clinical diagnosis of sinusitis and recovered viruses (rhinovirus, parainfluenza and influenza) out of $10.5 \%(11 / 105)$ of the specimens [6]. Another study recovered rhinovirus in punctures of the maxillary antrum from two out of 24 patients (8.34\%) evaluated with acute sinusitis [7].

Among ICU patients, ostium blockage and mucosal edema due to the presence of foreign bodies (i.e. various tubes) in the nasal cavity predispose to infection [3]. The supine position of the patient contributed with diminished mucociliary clearance and poor drainage of the paranasal sinuses [3].

Rouby et al. observed that after 7 days of nasotracheal intubation and nasogastric tube placement, 95\% of ICU patients demonstrated radiological pansinusitis, whereas after 7 days of orotracheal intubation and orogastric tube placement only $25 \%$ of them had evidence of radiological pansinusitis [8]. This study, conducted in mechanically ventilated patients, has at least four independent risk factors for sinusitis: the presence of a nasotracheal tube; the presence of a nasogastric tube; the duration of endotracheal intubation; and the duration of gastric aspiration [8].

Another factor that facilitates respiratory infections in ICU patients is the use of acid-suppressive drugs, proton-pump inhibitors or $\mathrm{H}_{2}$-receptor antagonists. These drugs bring bacterial and viral overgrowth in the stomach and gastric mucosa [9]. This association was reported by Hayase et al., who detected Influenza B virus genes in biopsy fragments of gastric mucosa in $46.25 \%$ of patients with esophageal and gastric diseases [10].

Patients with HIV/AIDS often suffer from airway infections and sinusitis is common in this patient population. One explanation for these observations is the reduction of nasal nitric oxide release in patients with HIV infection and impaired mucociliary function [11]. Other factors that predispose to rhinosinusitis in this patient population are the proliferation of lymphatic tissue due to polyclonal B-cell expansion, which contributes to nasal or sinus obstruction, and dysregulation of immunoglobulin A, E and G production [2].

The main etiologic agents of acute rhinosinusitis are the respiratory viruses, Streptococcus pneumoniae and Haemophilus influenzae [12]. In patients with HIV infection, Staphylococcus aureus and Pseudomonas aeruginosa can also cause rhinosinusitis [2; 12]. In hospitalized patients in particular, nasogastric or nasotracheal tubes predispose to sinusitis caused by Staphylococcus aureus and Pseudomonas aeruginosa [12].

Rhinoviruses are the respiratory viruses which are most frequently detected in sinus aspirates of patients with acute community-acquired sinusitis, and were recovered in $7 \%$ to $40 \%$ of the samples [13]. The use of RT-PCR enhanced the detection of rhinovirus infection [13]. Other viruses implied as the cause of rhinosinusitis were influenza virus, parainfluenza virus and adenovirus [3,12].

The combination of viral and bacterial infections is not uncommon, due to the physiopathological processes described above. Moreover, Jiang et al. have shown that RSV enhances attachment of Haemophilus influenzae to epithelial cells, a process largely mediated by Haemophilus influenzae P5-fimbria-binding receptors [14]. It is probable that similar mechanisms can occur between viruses and other bacteria.

In this case report, the patient had different bacterial infections, including rhinosinusitis, during her ICU stay. We identified RSV in maxillary sinus aspirate fourteen days after diagnosis in nasopharyngeal lavage. The presence of RSV in maxillary sinus may have contributed to bacterial superinfection.

Hall et al. reported that the duration of RSV shedding had a direct relation with the severity of the viral infection in children, supporting the observation of prolonged RSV shedding in the immunocompromised host [15].

Although little investigated in ICU patients, the present case report suggests that respiratory viruses can play a pathogenic role in airway infection and bring secondary bacterial overgrowth. More studies are necessary to better evaluate the role of RV infections in the pathogenesis and morbidity of upper and lower respiratory tract infections in ICU patients.

\section{References}

1. Lindbaek M. Acute Sinusitis: Guide to Selection of Antibacterial Therapy. Drugs 2004;64(8):805-19.

2. Gurney T.A., Lee K.C., Murr A.H. Contemporary issues in rhinosinusitis and HIV infection. Curr Opin Otolaryngol Head Neck Surg 2003;11(1):45-8.

3. Osguthorpe J.D., Hadley J.A. Rhinosinusitis: current concepts in evaluation and management. Med Clin North Am 1999;83(1):2741.

4. Gwaltney J.M. Jr. Acute community-acquired sinusitis. Clin Infect Dis 1996;23(6):1209-23.

5. Osur S.L. Viral respiratory infections in association with asthma and sinusitis: a review. Ann Allergy Asthma Immunol 2002;89(6):553-60.

6. Hamory B.H., Sande M.A., Sydnor A. Jr., et al. Etiology and antimicrobial therapy of acute maxillary sinusitis. J Infect Dis 1979;139(2):197-202.

7. Evans F.O. Jr., Sydnor J.B., Moore W.E., et al. Sinusitis of the maxillary antrum. N Engl J Med 1975;293(15):735-9.

8. Rouby J.J., Laurent P., Gosnach M., et al. Risk factors and clinical relevance of nosocomial maxillary sinusitis in the critically ill. Am J Respir Crit Care Med 1994;150(3):776-83.

9. Laheij R.J., Van Ijzendoorn M.C., Janssen M.J., Jansen J.B. Gastric acid-suppressive therapy and community-acquired respiratory infections. Aliment Pharmacol Ther 2003;18(8):847-51.

10. Hayase Y., Tobita K., Sato H. Detection of type B influenza virus genes from biopsied gastric mucosa. J Gastroenterol 2002;37(2):101-5.

11. Palm J., Lidman C., Graf P., et al. Nasal nitric oxide is reduced in patients with HIV. Acta Otolaryngol 2000;120(3):420-3. 
12. Sociedad Espanola de Quimioterapia; Sociedad Espanola de Otorrinolaringologia y Patologia Cervico-Facial. [Diagnosis and antimicrobial treatment of sinusitis] [Article in Spanish] Rev Esp Quimioterap 2003;16(2):239-51.

13. Pitkaranta A., Arruda E., Malmberg H., Hayden F.G. Detection of rhinovirus in sinus brushings of patients with acute communityacquired sinusitis by reverse transcription-PCR. J Clin Microbiol 1997;35(7):1791-3.
14. Jiang Z., Nagata N., Molina E., et al. Fimbria-mediated enhanced attachment of nontypeable Haemophilus influenzae to respiratory syncytial virus-infected respiratory epithelial cells. Infect Immun 1999;67(1):18792.

15. Hall C.B., Douglas R.G. Jr., Geiman J.M. Respiratory syncytial virus infections in infants: quantitation and duration of shedding. J Pediatr 1976;89(1):11-5. 